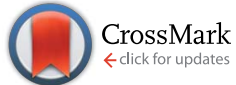

Cite this: RSC Adv., 2015, 5, 88298

Received 7th September 2015 Accepted 9th October 2015

DOI: $10.1039 / c 5 r a 18236 k$

www.rsc.org/advances

\section{Influence of facets and heterojunctions in photoactive bismuth oxyiodide $\uparrow$}

\author{
Aijuan Han, ${ }^{\text {ab }}$ Jiulong Sun, ${ }^{\text {ab }}$ Xuanhao Lin, ${ }^{\text {a }}$ Cheng-Hui Yuan, ${ }^{a}$ Gaik Khuan Chuah*a \\ and Stephan Jaenicke*a
}

The role of facetted crystalline nanosheets and heterojunctions in bismuth oxyiodide composites for the photodegradation of phenolic compounds is investigated. Single-crystalline nanosheets with either $\{001\}$ or $\{110\}$ planes as the dominant facets were formed by tuning the $\mathrm{pH}$ during synthesis. The $\{001\}$ facetted $\mathrm{BiOl}$ consisted of large crystallites with low surface areas in contrast to the thinner and smaller $\{110\}$-facetted platelets. The specific activity per $\mathrm{m}^{2}$ for the photodegradation of $p$-cresol was $\sim 5$ times higher over the $\{001\}$ than over the $\{110\}$-facetted $\mathrm{BiOl}$ which can be correlated to the stronger adsorption of the substrate at the oxygen-rich $\{001\}$ surface. However, the low surface area of $<1 \mathrm{~m}^{2} \mathrm{~g}^{-1}$ is a drawback for practical use. Instead, by simple calcination of $\{110\}$-facetted $\mathrm{BiOl}$ at $350{ }^{\circ} \mathrm{C}$, composites of different $\mathrm{Bi}_{x} \mathrm{O}_{y} \mathrm{l}_{z}$ phases with closely related crystalline structures were formed. The intimate contact between the phases results in the formation of heterojunctions which greatly improve the photoactivity, even over that of $\{001\}$-facetted $\mathrm{BiOI}$. The most active photocatalyst was a composite of $\left.\mathrm{Bi}_{7} \mathrm{O}_{9}\right|_{3} / \alpha-\mathrm{Bi}_{5} \mathrm{O}_{7} /$ which maintained its activity in the presence of chloride and nitrate anions and was recyclable. Scavenger studies revealed that holes were active species for the degradation of $p$-cresol.

\section{Introduction}

The activity of a photocatalyst depends on a number of factors including band gap and surface area. While the width of the band gap affects the optical absorption characteristics, the absolute band position (referenced against the Fermi level or the potential of the normal hydrogen electrode) determines the reduction and oxidation abilities. ${ }^{\mathbf{1}} \mathrm{A}$ higher surface area is usually beneficial as more active surface sites are available. ${ }^{2}$ However, for a nonporous material, a higher surface area means smaller particle size which in turn could increase the rate of surface electron/hole recombination, thus offsetting the benefits of the high surface area. Hence there is an optimum particle size for photocatalysts. ${ }^{3}$ Furthermore, different crystal facets possess particular electronic and geometric structures that offer distinctive photocatalytic activities. Anatase titania with $\{001\}$ facets has been found to be more reactive than titania with $\{101\}$ facets due to differences in surface energy. ${ }^{4-6}$ Jiang et al. reported that single-crystalline nanosheets (SCNs) of BiOCl with predominantly exposed $\{001\}$ facets exhibited high activity for the direct semiconductor photoexcitation leading to pollutant

${ }^{a}$ Department of Chemistry, National University of Singapore, 3 Science Drive, Singapore 117543, Singapore.E-mail: chmcgk@nus.edu.sg; chmsj@nus.edu.sg; Fax: +65 6779 1691; Tel: +65 6516 2839, +65 65162918

${ }^{b}$ NUS Environmental Research Institute, National University of Singapore, $5 A$ Engineering Drive 1, \#02-01, Singapore 117411, Singapore

$\dagger$ Electronic supplementary information (ESI) available. See DOI: $10.1039 / \mathrm{c} 5 \mathrm{ra} 18236 \mathrm{k}$ degradation under UV light, while the material with exposed $\{010\}$ facets had higher activity for the indirect dye-sensitized photodegradation under visible light. ${ }^{7}$ Hence, the facetcontrolled fabrication of single-crystalline photocatalysts introduces another degree of freedom in the design of photocatalysts. $^{\mathbf{8 - 1 2}}$

BiOI has a tetragonal PbFCl (matlockite) structure where $\left[\mathrm{Bi}_{2} \mathrm{O}_{2}\right]$ slabs are interleaved between double layers of iodine ions (Fig. 1a). The electrons in the $5 \mathrm{~s}$ orbital of the iodine ions are easily excited, so that the band gap of BiOI is only $\sim 1.8 \mathrm{eV}$. Hence, it can utilize a large proportion of the visible light spectrum. BiOI in different morphologies such as nanoparticles, ${ }^{\mathbf{1 3 , 1 4}}$ nanobelts, ${ }^{15}$ nanoplates, ${ }^{16,17}$ and $3 \mathrm{D}$ microspheres ${ }^{18-21}$ has been reported as
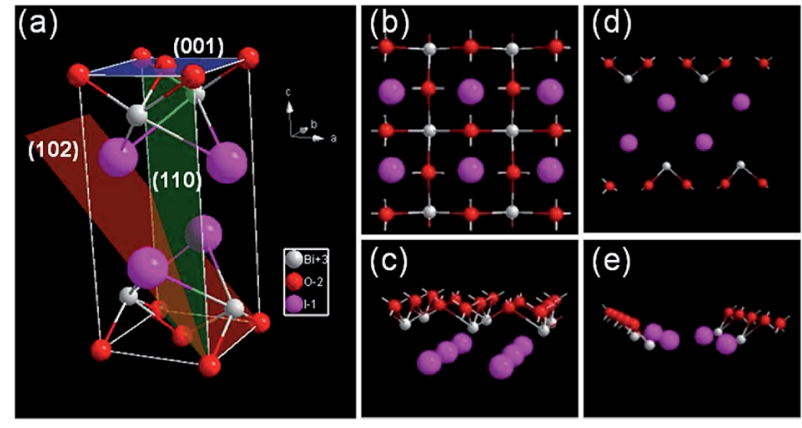

Fig. 1 (a) Crystal structure of $\mathrm{BiOl}$, ( $\mathrm{b}$ and d) top and (c and e) side views of the atomic arrangement in the (001) and (110) facets. 

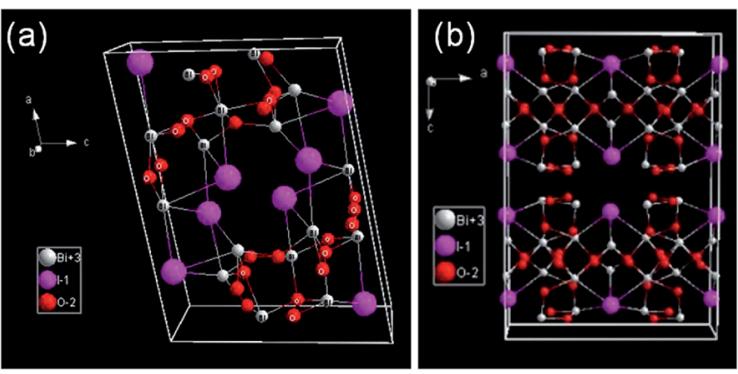

Fig. 2 Crystal structure of (a) $\mathrm{Bi}_{4} \mathrm{O}_{5} \mathrm{I}_{2}$ and (b) $\alpha-\mathrm{Bi}_{5} \mathrm{O}_{7} \mathrm{I}$.

photocatalyst for the decomposition of various organic pollutants such as Rhodamine B, methyl orange, and phenol. Studies on BiOI single crystalline nanosheets prepared by annealing $\mathrm{BiI}_{3}$ in air showed that the $\{001\}$ facets are most reactive. ${ }^{22}$

In view of these reports, we were interested in the factors that influence the photoactivity of BiOI such as morphology, surface area and heterojunctions. We adjusted the synthesis $\mathrm{pH}$ to direct the facet formation in BiOI, similar to that reported for BiOCl. $^{7}$ The density of oxygen atoms in the (001) and (110) planes differs, being 6.27 and 1.94 atoms per $\mathrm{nm}^{2}$, respectively (Fig. 1). Under acidic conditions, $\mathrm{H}^{+}$will be adsorbed more strongly on the O-terminated (001) than on the (110) surface. Consequently, growth in the [001] direction is hindered, leading to predominance of (001) facetted planes. At higher $\mathrm{pH}$, this hindrance is reduced due to the lower $\mathrm{H}^{+}$concentration, and other slow growing planes become predominant.

To form heterojunctioned composites, we took advantage of the fact that the iodine atoms in the BiOI structure can be easily replaced by oxygen atoms upon thermal treatment, resulting in various oxygen-rich bismuth oxyiodides, namely, $\mathrm{Bi}_{4} \mathrm{O}_{5} \mathrm{I}_{2}$, $\mathrm{Bi}_{7} \mathrm{O}_{9} \mathrm{I}_{3}$, and $\alpha$ - and $\beta-\mathrm{Bi}_{5} \mathrm{O}_{7} \mathrm{I}^{23-28}$ These bismuth oxyiodides share similar layered $[\mathrm{BiO}]^{+}$structures but differ in the halogen content of the sandwich layer. In $\mathrm{Bi}_{4} \mathrm{O}_{5} \mathrm{I}_{2},[\mathrm{BiO}]^{+}$layers

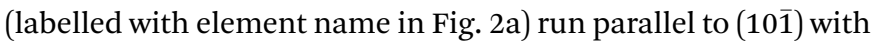
$\left[\mathrm{BiO}_{2}\right]^{-}$chains parallel to [010] attached on both sides. The $\left[\mathrm{Bi}_{4} \mathrm{O}_{5}\right]^{2+}$ layers formed are separated from each other by layers of halogen ions. In $\alpha-\mathrm{Bi}_{5} \mathrm{O}_{7} \mathrm{I},\left[\mathrm{Bi}_{2} \mathrm{O}_{2}\right]^{2+}$ sheets aligned parallel to (001) are mutually connected by folded 1-dimensional $\left[\mathrm{Bi}_{4} \mathrm{O}_{8}\right]^{4-}$ ribbons with axes parallel to $[010]$ and oriented parallel to (100) (Fig. 2b). We envision that the similarity of the crystal structures of the various bismuth oxyiodides should enable the formation of intimate junctions between the band structures in the crystalline domains in the $\mathrm{Bi}_{x} \mathrm{O}_{y} \mathrm{I}_{z}$ composites, which at the right energy levels should facilitate electron-hole separation.

\section{Experimental}

\subsection{Preparation of BiOI}

Typically, $10 \mathrm{mmol}$ of $\mathrm{KI}$ in $6 \mathrm{ml}$ deionized water was added with stirring to a solution with $2 \mathrm{mmol}$ of $\mathrm{Bi}\left(\mathrm{NO}_{3}\right)_{3} \cdot 5 \mathrm{H}_{2} \mathrm{O}$ in $21 \mathrm{ml}$ deionized water, acidified with $1.4 \mathrm{ml}$ glacial acetic acid. The required amount of $1 \mathrm{M} \mathrm{NaOH}$ solution was added to adjust the $\mathrm{pH}$ of the solution to $x(x=3,4,5,6,7,8)$. After stirring for 30 min, the solution was poured into a $40 \mathrm{ml}$ Teflon-lined stainless steel autoclave and kept at $160{ }^{\circ} \mathrm{C}$ for $2 \mathrm{~h}$ under autogenous pressure. The resulting precipitate was collected by centrifugation, washed with absolute ethanol, and dried in an oven at $80{ }^{\circ} \mathrm{C}$ overnight. The product obtained was denoted as BiOI$\mathrm{pH} x$. The $\mathrm{pH}$ of the suspension without adding any $\mathrm{NaOH}$ solution was 2.3, therefore this sample was denoted as BiOIpH2.3.

\subsection{Synthesis of bismuth oxyiodide heterojunctions}

The as-prepared BiOI-pH6 was calcined at 300 to $400{ }^{\circ} \mathrm{C}$ in air (1-5 h, $1{ }^{\circ} \mathrm{C} \min ^{-1}$ ramp) using a Nabertherm oven. The products obtained are denoted as BiOI-pH6- $y$ - $z$ where $y$ represents the calcination temperature and $z$ denotes the calcination time.

\subsection{Characterization}

Powder X-ray diffraction patterns were measured with a Siemens D5005 diffractometer using $\mathrm{Cu} \mathrm{K}_{\alpha}$ radiation for $2 \theta$ from $5-80^{\circ}$ at $0.02^{\circ} \mathrm{s}^{-1}$ and a dwell time of $1 \mathrm{~s}$ per step. Nitrogen adsorption and desorption isotherms were obtained at $-196{ }^{\circ} \mathrm{C}$ using a TriStar 3000 Micromeritics porosimeter. The specific surface area was calculated using the BET (Brunauer-EmmettTeller) equation. Scanning electron microscopy (SEM) was performed on a JEOL JSM-6701F SEM (field-emission) microscope with $5 \mathrm{kV}$ electron beam energy. The sample was spread on a graphite adhesive tape and coated with $\sim 2 \mathrm{~nm}$ Pt. Transmission electron micrographs were obtained with a JEOL 3010 operated at $200 \mathrm{kV}$. Thermogravimetric measurements were carried out using a TA Instruments SDT 2960 simultaneous DTA-TGA. Diffuse reflectance spectra were measured with a Shimadzu UV-2450 UV-Visible spectrophotometer.

\subsection{Photocatalytic activity}

A suspension of $0.10 \mathrm{~g}$ photocatalyst in $100 \mathrm{ml}$ of an aqueous solution of $p$-cresol (24 ppm) was magnetically stirred for $1 \mathrm{~h}$ to establish an adsorption-desorption equilibrium. An aliquot was removed to determine the $p$-cresol concentration in the solution before irradiation by a $22 \mathrm{~W}$ fluorescent lamp $\left(3.3 \times 10^{16}\right.$ photons per s), placed $1 \mathrm{~cm}$ at the side of reactor. After specific time intervals, $2 \mathrm{ml}$ aliquots were withdrawn and centrifuged at $6000 \mathrm{rpm}$ for $5 \mathrm{~min}$ to remove any suspended particles. The concentration of $p$-cresol was determined from the absorption band at $\lambda \sim 277.5 \mathrm{~nm}$ using a UV-vis spectrophotometer (Shimadzu, UV-1601). The degradation efficiency was calculated as: $\mathrm{DE}=100 \% \times\left(C_{0}-C\right) / C_{0}$, where $C_{0}$ is the concentration of $p$ cresol after equilibration in the dark, and $C$ is the $p$-cresol concentration at time $t$. Photoactivity tests for other phenolic substrates were also carried out at $24 \mathrm{ppm}$ concentration. The used catalyst was recovered by centrifugation, washed with deionized water, recalcined at $300{ }^{\circ} \mathrm{C}$ for $1 \mathrm{~h}$ and tested in further runs. To test the influence of other ions, the activity was also evaluated in the presence of $1 \mathrm{mM}$ ammonium nitrate and sodium chloride. The total organic carbon (TOC) was measured with a Shimadzu TOC-VWS Total Organic Carbon Analyzer.

To detect intermediates of the $p$-cresol degradation, $20 \mathrm{ml}$ of a $p$-cresol solution with concentration of $2.4 \mathrm{~g} \mathrm{~L} \mathrm{~L}^{-1}$ was 
irradiated for $19 \mathrm{~h}$ in the presence of $0.4 \mathrm{~g}$ BiOI-pH6-350-3 under the same lamp. Aliquots were removed at different time intervals and analyzed on a Dionex Ultimate 3000 RSLC system coupled to an AmaZon X system (LC/MS). The column was a Luna $\mathrm{C} 18$, and atmospheric pressure chemical ionization (APCI) was used with full scan for negative ions from 70 to 500 amu. The mobile phase was $30 \mathrm{vol} \%$ acetonitrile in water.

The nature of the active species was determined by investigating the photocatalytic degradation of $p$-cresol in the presence of various scavengers, i.e., $\mathrm{K}_{2} \mathrm{C}_{2} \mathrm{O}_{4}, t$-butanol and $\mathrm{KBrO}_{3}$. The procedure was the same as in the activity test except that a fivefold molar excess (relative to $p$-cresol) of the scavenger was added together with the photocatalyst. The presence of ${ }^{\circ} \mathrm{OH}$ radicals was deduced by trapping with terephthalic acid. Terephthalic acid itself does not fluoresce, but upon reaction with 'OH radicals, 2-hydroxyterephthalic acid is formed which has a broad emission band with maximum at $425 \mathrm{~nm}$. About $0.1 \mathrm{~g}$ of the photocatalyst was added to a $200 \mathrm{ml}$ solution containing terephthalic acid $(16.6 \mathrm{mg})$ in $2 \mathrm{mM} \mathrm{NaOH}$. After irradiation with the $22 \mathrm{~W}$ fluorescent lamp, aliquots were removed at $30 \mathrm{~min}$ interval, centrifuged and the fluorescent emission spectrum of the solution was measured by a Gilden FluoroSNSfluorimeter using an excitation wavelength of $315 \mathrm{~nm}$.

\section{Result and discussion}

\subsection{Characterization of BiOI synthesized at different $\mathbf{p H}$}

The X-ray diffractograms of BiOI synthesized between $\mathrm{pH} 2.3$ to 7 were purely tetragonal (JCPDS 10-0045) (Fig. 3). Bismuth oxyiodide formed at $\mathrm{pH} 2.3$ had diffraction peaks with high intensity especially those of the $(00 n)$ planes. The intensity of the $(00 n)$ peaks fell drastically in samples synthesized at higher $\mathrm{pH}$ but the (102) and (110) peak intensities remained unchanged. For $\mathrm{pH} 5-8$, the (110) peak intensity was increased relative to the (102), indicating a predominance of this facet. The sample synthesized at $\mathrm{pH} 8$ contained BiOI as well as $\mathrm{Bi}_{4} \mathrm{O}_{5} \mathrm{I}_{2}$. The latter was identified from peaks at $2 \theta \sim 8.6^{\circ}$ and $28.6^{\circ}$ (JCPDS 12-1474). At this high $\mathrm{pH}, \mathrm{Bi}_{4} \mathrm{O}_{5} \mathrm{I}_{2}$ can form by the partial substitution of $\mathrm{I}^{-}$by $\mathrm{OH}^{-}\left(4 \mathrm{BiOI}+2 \mathrm{OH}^{-} \rightarrow \mathrm{Bi}_{4} \mathrm{O}_{5} \mathrm{I}_{2}+\right.$ $\left.2 \mathrm{I}^{-}+\mathrm{H}_{2} \mathrm{O}\right)$.

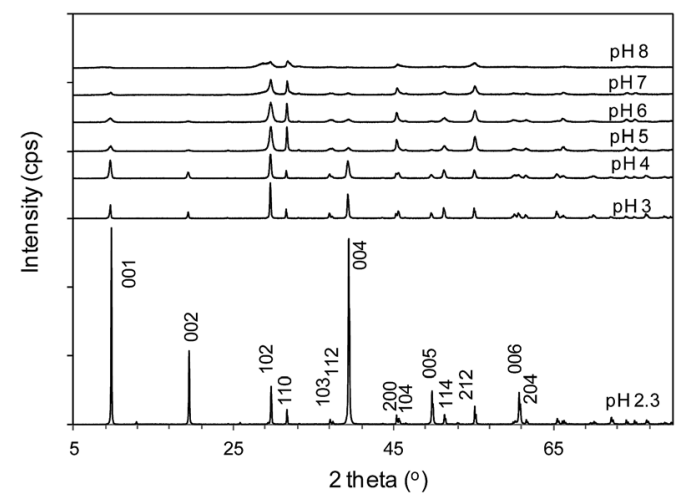

Fig. 3 X-ray diffraction patterns of $\mathrm{BiOl}$ samples prepared at different $\mathrm{pH}$.
The crystallite size was calculated from the width of the (001) peak using the Scherer equation (Table 1). The crystallite size decreased from 126 to $16 \mathrm{~nm}$ as the synthesis $\mathrm{pH}$ rose from 2.3 to 7. This can be attributed to the decreasing solubility of BiOI with $\mathrm{pH}$ so that upon mixing of the reactants, precipitation occurs more rapidly and smaller crystallites form.

Scanning electron microscopy (SEM) showed that irrespective of the synthesis $\mathrm{pH}$, all the BiOI samples had a platelike morphology (Fig. 4). With increasing $\mathrm{pH}$, the lamellae became smaller and thinner (Table 1). This change in morphology is reflected by an increase of surface area and the decrease in X-ray peak intensity. BiOI synthesized between $\mathrm{pH}$ 2.3 to 4 had surface areas of 0.65 to $3.98 \mathrm{~m}^{2} \mathrm{~g}^{-1}$ while materials synthesized at pH 5 to 8 had surface areas of 9.8 to $23.9 \mathrm{~m}^{2} \mathrm{~g}^{-1}$. The $\{001\}$ facets were dominant in the transmission electron micrographs of BiOI synthesized below pH 4. For BiOI-pH2.3, lattice fringes with an interplanar spacing of $0.282 \mathrm{~nm}$ corresponding to the $\{110\}$ atomic planes could be seen (Fig. 5). The sharp spots in the selected-area electron diffraction (SAED) pattern indicate the single crystalline nature. The labelled angle of $45^{\circ}$ corresponds to that between the (110) and (200) planes for the tetragonal symmetry group, thus indexing the diffraction spots to the [001] zone axis. Therefore, the bottom and top surfaces of the BiOI-pH2.3 sample can be assigned to the $\{001\}$ facets, which agrees well with the observed reflexes in XRD.

In contrast, the SAED pattern for BiOI synthesized at a higher $\mathrm{pH}$ of 6 shows the angle between the diffraction spots to be $42.8^{\circ}$ (Fig. 6). This value corresponds to the angle between the (113) and (110) planes. The set of diffraction spots can therefore be indexed as the [110] zone axis of tetragonal BiOI so that the exposed facets are identified as those of $\{110\}$. Hence, by varying the $\mathrm{pH}$ during synthesis, it is possible to change the dominant exposed facets from (001) to (110).

The samples absorb in the visible range up to $670 \mathrm{~nm}$ (Fig. S1 $\dagger$ ). The band gap energy was estimated from the intercept of a $\left(F\left(R_{\infty}\right) h \nu\right)^{1 / \eta}$ versus $h \nu$ plot, where $F\left(R_{\infty}\right)=\left(1-R_{\infty}\right)^{2} /$ $2 R_{\infty}, R_{\infty}=$ the reflectance of the sample using $\mathrm{BaSO}_{4}$ as a reference, $\nu=$ light frequency, $E_{\mathrm{g}}=$ band-gap energy, and $\eta=$ 2 for an indirect transition. ${ }^{29}$ The band gap increased slightly from 1.72 to $1.94 \mathrm{eV}$ with higher $\mathrm{pH}$ during synthesis (Table 1).

Table 1 Physical properties of $\mathrm{BiOl}$ samples synthesized at different $\mathrm{pH}$

\begin{tabular}{llllll}
\hline \multicolumn{2}{c}{ XRD } & \multicolumn{3}{l}{} & \\
\cline { 3 - 4 } & & & $\begin{array}{l}\text { Crys. size } \\
(\mathrm{nm})\end{array}$ & $\begin{array}{l}\text { Surf. area } \\
\left(\mathrm{m}^{2} \mathrm{~g}^{-1}\right)\end{array}$ & $E_{\mathrm{g}}(\mathrm{eV})$ \\
\hline $\mathrm{pH}$ & $(001) /(102)$ & $(110) /(102)$ & & 0.65 & 1.72 \\
2.3 & 3.52 & 0.31 & 126 & 2.03 & 1.84 \\
3 & 0.39 & 0.20 & 60.1 & 3.98 & 1.86 \\
4 & 0.18 & 0.18 & 36.9 & 9.81 & 1.88 \\
5 & 0.24 & 0.43 & 25.7 & 15.3 & 1.90 \\
6 & 0.24 & 0.46 & 20.3 & 19.1 & 1.92 \\
7 & 0.13 & 0.47 & 16.2 & 23.9 & 1.94 \\
8 & 0.04 & 0.58 & - & &
\end{tabular}



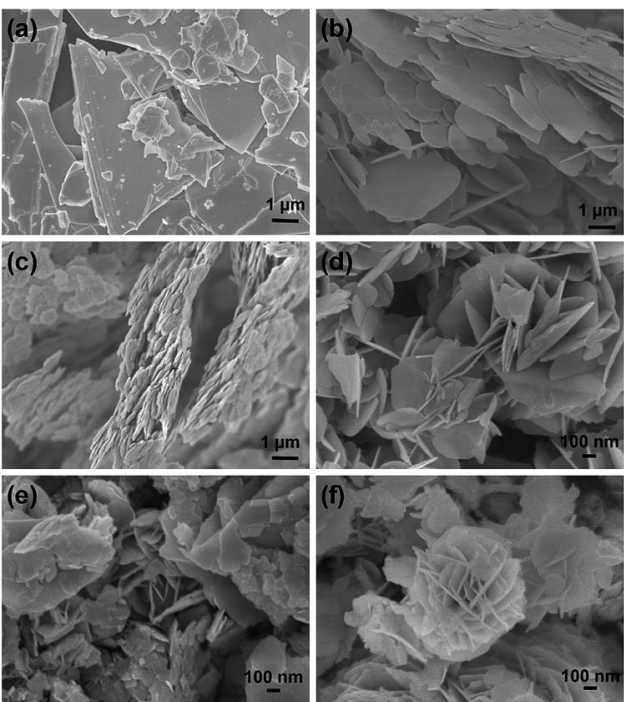

Fig. 4 SEM images of (a) BiOl-pH2.3, (b) BiOl-pH3, (c) BiOl-pH4, (d) $\mathrm{BiOl}-\mathrm{pH} 6$, (e) $\mathrm{BiOl}-\mathrm{pH} 7$, and (f) $\mathrm{BiOl}-\mathrm{pH} 8$.
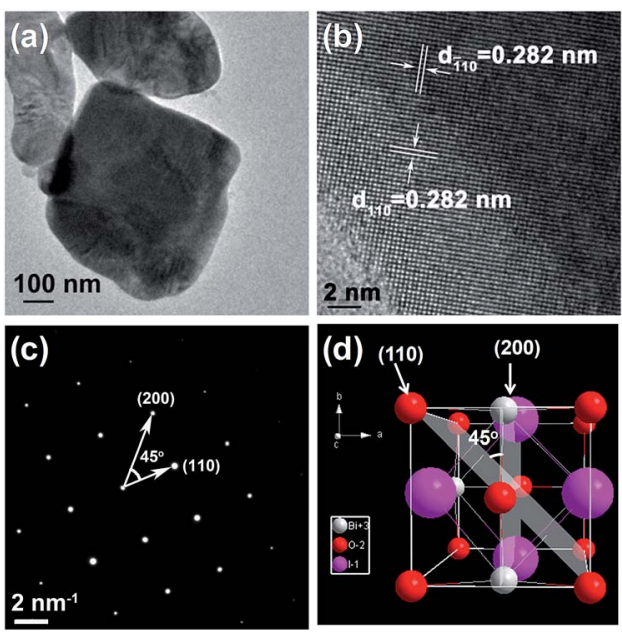

(e)

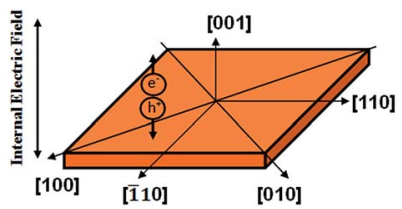

Fig. 5 (a) TEM image, (b) HR-TEM image, (c) SAED pattern, (d) top view of the (001) facet and (e) crystallographic directions for BiOl-pH2.3 SCN.

This increase is reflected by a change from dark red in BiOIpH2.3 to orange for BiOI-pH8 (Fig. S2 $\dagger$ ).

\subsection{Photocatalytic activity of BiOI with different exposed facets}

The photocatalytic activity of BiOI synthesized at different $\mathrm{pH}$ was assessed using $p$-cresol $\left(\mathrm{CH}_{3}\left(\mathrm{C}_{6} \mathrm{H}_{4}\right) \mathrm{OH}\right)$ as a model pollutant. In the absence of the photocatalyst, there was almost
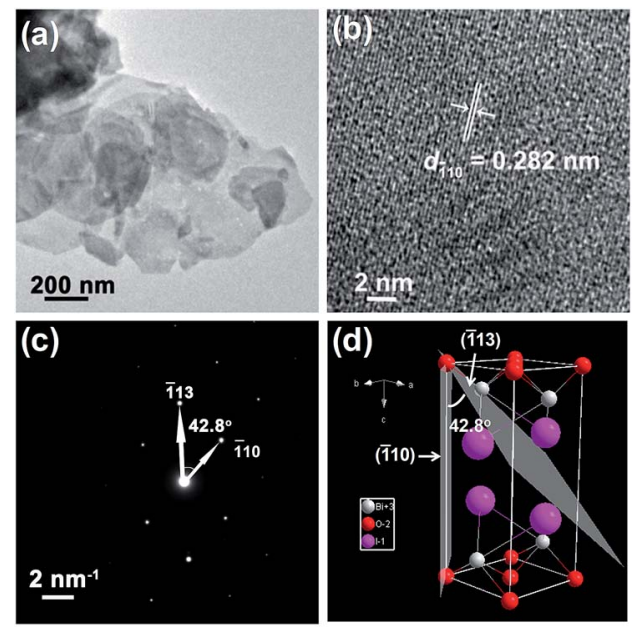

(e)

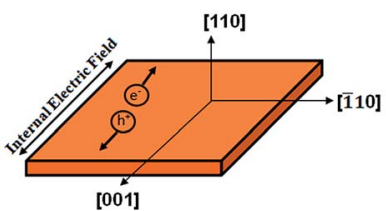

Fig. 6 (a) TEM image, (b) HR-TEM image, (c) SAED pattern, (d) top view of the (110) facet and (e) crystallographic directions for BiOI-pH6 SCN.

no degradation of $p$-cresol under visible light irradiation (Fig. 7). The degradation efficiency can be correlated with the $\mathrm{pH}$ during synthesis: it increased to a maximum at $\mathrm{pH} 6$ before decreasing with higher $\mathrm{pH}$. However, BiOI synthesized at $\mathrm{pH} 7$ and 8 were significantly more active than the materials prepared at acidic pH. Because the photodegradation normally takes place at the surface of the catalyst, surface structure and surface area will significantly influence the photoactivity. Pseudo firstorder kinetics were applied to $p$-cresol degradation according to the equation: $\ln \left(C_{0} / C\right)=k t$ where $k$ is the pseudo-first-order rate constant (Fig. $\mathrm{S} 3 \dot{\dagger})$. The rate constant was normalized to the catalyst weight $\left(k_{\mathrm{W}}\right)$ as well as the surface area $\left(k_{\mathrm{SA}}\right)$ to allow for a comparison of the activity (Table 2).

Interestingly, $k_{\mathrm{W}}$ and $k_{\mathrm{SA}}$ show different trends for BiOI synthesized at different $\mathrm{pH}$. The rate constant per gram catalyst,

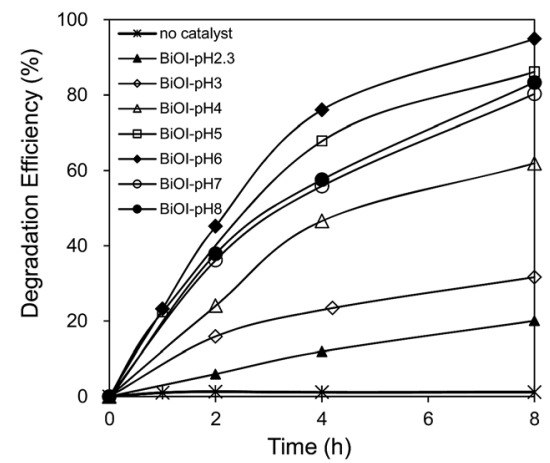

Fig. 7 Photocatalytic activity of BiOl for $p$-cresol degradation under visible light. 
Table 2 Rate constant and $p$-cresol adsorption over BiOl prepared at different $\mathrm{pH}$

\begin{tabular}{llll}
\hline $\mathrm{pH}$ & $k_{\mathrm{W}}\left(\mathrm{h}^{-1} \mathrm{~g}^{-1}\right)$ & $k_{\mathrm{SA}}\left(\mathrm{h}^{-1} \mathrm{~m}^{-2}\right)$ & $C_{\mathrm{ads}}\left(\mathrm{mg} \mathrm{m}^{-2}\right)$ \\
\hline 2.3 & 0.28 & 0.43 & 0.44 \\
3 & 0.68 & 0.33 & 0.33 \\
4 & 1.25 & 0.31 & 0.30 \\
5 & 2.55 & 0.26 & 0.06 \\
6 & 3.65 & 0.24 & 0.05 \\
7 & 2.05 & 0.11 & 0.03 \\
8 & 2.23 & 0.09 & 0.02
\end{tabular}

$k_{\mathrm{W}}$, increased 13-fold from $0.28 \mathrm{~h}^{-1} \mathrm{~g}^{-1}$ at $\mathrm{pH} 2.3$ to $3.65 \mathrm{~h}^{-1} \mathrm{~g}^{-1}$ at pH 6 before decreasing to $\sim 2.1 \mathrm{~h}^{-1} \mathrm{~g}^{-1}$ for yet higher $\mathrm{pH}$. On the other hand, the rate constant normalized by surface area, $k_{\mathrm{SA}}$, decreased from $0.43 \mathrm{~h}^{-1} \mathrm{~m}^{-2}$ at $\mathrm{pH} 2.3$ to $0.09 \mathrm{~h}^{-1} \mathrm{~m}^{-2}$ at $\mathrm{pH} 8$, showing that the intrinsic activity was higher for the $\{001\}$ dominated facets. To obtain an insight into the facet-dependent photocatalytic activity, we examined the adsorption capacities of $p$-cresol on the BiOI samples (Table 2). The samples formed at low $\mathrm{pH}$ with dominant $\{001\}$ facets adsorb $0.30-0.44 \mathrm{mg} p$-cresol $\mathrm{m}^{-2}$ as compared to only $0.02-0.06 \mathrm{mg} \mathrm{m}^{-2}$ for materials formed at $\mathrm{pH} 5-8$. The former values correspond to a complete monolayer based on an area of $0.385 \mathrm{~nm}^{2}$ for the $p$-cresol molecule, as obtained from the density of the liquid assuming close packing of spherical molecules. The preferential surface coverage can be attributed to the high density of oxygen atoms at the $\{001\}$ planes which favours the adsorption of $p$-cresol through hydrogen bonding with its hydroxyl group. The layered structure of BiOI leads to a permanent dipole between the $\left[\mathrm{Bi}_{2} \mathrm{O}_{2}\right]^{2+}$ and $\mathrm{I}^{-}$slabs, resulting in the generation of an internal electric field. Photon absorption creates an electron-hole pair in the bulk of the crystal. The electron-hole pairs are separated under the influence of this internal electric field. Because this internal field is perpendicular to the exposed facets in the $\{001\}$ oriented crystals (Fig. 5e) but parallel in the $\{110\}$-facetted samples (Fig. 6e), the field supports diffusion of charges to the surface along the shortest crystal dimension in the $\{001\}$ dominant sample, leading to the higher $k_{\mathrm{SA}}$.

\subsection{Formation of heterojunctioned composites}

Although the intrinsic photoactivity of the $\{110\}$-dominant BiOIpH6 was only about half that of the $\{001\}$-facetted BiOI-pH2.3, it had a much higher surface area, 15.3 vs. $0.65 \mathrm{~m}^{2} \mathrm{~g}^{-1}$. This more than compensates for the photoactivity when used on an equal weight basis. We investigated whether calcination could further improve the activity. BiOI is stable up to $300{ }^{\circ} \mathrm{C}$, whereupon it slowly transforms into $\mathrm{Bi}_{4} \mathrm{O}_{5} \mathrm{I}_{2}$ (JCPDS 12-1474) with loss of iodine (Fig. $\mathrm{S} 4 \dagger$ ). After calcination at $300{ }^{\circ} \mathrm{C}$ for $1 \mathrm{~h}$, the diffraction peaks were shifted to smaller $2 \theta$ and broadened (Fig. 8 and $55 \dagger$ ). The peaks at $27.4^{\circ}$ and $31.6^{\circ}$ can be treated as characteristic peaks for $\mathrm{BiOI}$ and $\mathrm{Bi}_{4} \mathrm{O}_{5} \mathrm{I}_{2}$, respectively. From the peak areas, the proportion of $\mathrm{Bi}_{4} \mathrm{O}_{5} \mathrm{I}_{2} / \mathrm{BiOI}$ was $55: 45$ (Table 3). Composition changes calculated from the weight losses measured with thermogravimetry gave $\mathrm{Bi}_{4} \mathrm{O}_{5} \mathrm{I}_{2} / \mathrm{BiOI}$ of $59: 41$,

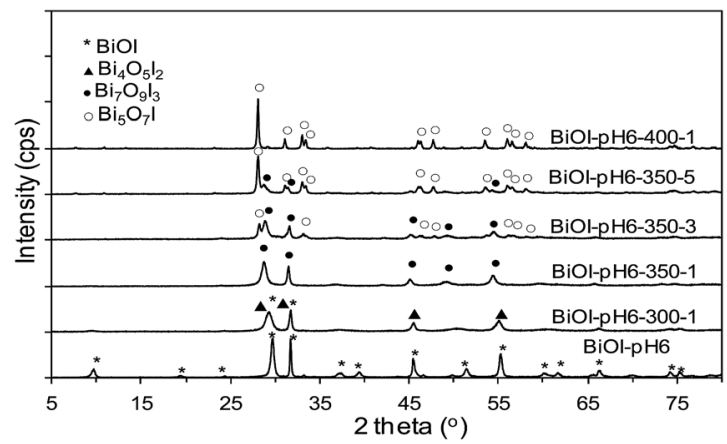

Fig. 8 X-ray diffractograms of as-synthesized and calcined $\mathrm{BiOl-pH6}$ samples.

in good agreement with the XRD results. An increase in the treatment temperature to $350{ }^{\circ} \mathrm{C}$ resulted in a shift of the diffraction lines to even smaller $2 \theta$, where the diffraction pattern indicated the presence of a new phase, $\mathrm{Bi}_{7} \mathrm{O}_{9} \mathrm{I}_{3}$. This is again corroborated by the weight loss upon heating the sample from 100 to $850{ }^{\circ} \mathrm{C}$ (Fig. S4 $\dagger$ ). The measured weight loss of $17.7 \%$ agrees closely with that calculated for the transformation of $\mathrm{Bi}_{7} \mathrm{O}_{9} \mathrm{I}_{3}$ to $\mathrm{Bi}_{2} \mathrm{O}_{3}(17.9 \%)$. Hence, it can be concluded that BiOIpH6-350-1 is pure $\mathrm{Bi}_{7} \mathrm{O}_{9} \mathrm{I}_{3}$. Extending the heating time at this temperature to $3 \mathrm{~h}$ resulted in the appearance of another bismuth oxyiodide compound, $\alpha-\mathrm{Bi}_{5} \mathrm{O}_{7} \mathrm{I}$ (JCPDS 40-0548) which coexisted with $\mathrm{Bi}_{7} \mathrm{O}_{9} \mathrm{I}_{3}$. From the peak areas, the proportion of $\mathrm{Bi}_{7} \mathrm{O}_{9} \mathrm{I}_{3} / \alpha-\mathrm{Bi}_{5} \mathrm{O}_{7} \mathrm{I}$ was $35: 65$ (Table 3). The proportion of $\alpha-\mathrm{Bi}_{5} \mathrm{O}_{7} \mathrm{I}$ increased to $74: 26$ when the heating time was extended to $5 \mathrm{~h}$. After treatment at an even higher temperature $\left(400{ }^{\circ} \mathrm{C}\right)$, only the pure $\alpha-\mathrm{Bi}_{5} \mathrm{O}_{7} \mathrm{I}$ phase was observed (Fig. 8). Thus, by varying the heating time at $350{ }^{\circ} \mathrm{C}$, compositions with different proportions of $\mathrm{Bi}_{7} \mathrm{O}_{9} \mathrm{I}_{3}$ and $\alpha-\mathrm{Bi}_{5} \mathrm{O}_{7} \mathrm{I}$ could be formed. Scanning electron micrographs revealed that the plate-like morphology of the material was not affected by the calcination (Fig. S6†).

Four distinct lattice spacings are observed in the HRTEM of BiOI-pH6-350-3 (Fig. 9b). The lattice spacing of $0.288 \mathrm{~nm}$ can be uniquely assigned to the (311) lattice plane of $\alpha-\mathrm{Bi}_{5} \mathrm{O}_{7} \mathrm{I}$ while the others at $0.310,0.403$ and $0.668 \mathrm{~nm}$ match $d$-spacings in $\mathrm{Bi}_{7} \mathrm{O}_{9} \mathrm{I}_{3}$. These results confirm that the thermal treatment leads to $\mathrm{Bi}_{7} \mathrm{O}_{9} \mathrm{I}_{3}$ and $\alpha-\mathrm{Bi}_{5} \mathrm{O}_{7} \mathrm{I}$ phases that are intimately mixed in the nanosized range.

The UV-visible diffuse reflectance spectra of the calcined samples showed a blue shift that depended on the temperature and length of calcination (Fig. S7 $\dagger$ ). The transformation of BiOI to $\alpha-\mathrm{Bi}_{5} \mathrm{O}_{7} \mathrm{I}$ is accompanied by a shift in the absorption edge from $\sim 620$ to $\sim 460 \mathrm{~nm}$ as the color of the sample changed from dark orange to pale yellow (Fig. S8 $\dagger$ ). The band gap $E_{\mathrm{g}}$ increased from 1.90 to $2.58 \mathrm{eV}$ for the composites. The electronic structure was calculated by density functional theory using CASTEP code (Fig. S9†). The valence band in BiOI is composed mainly of iodine $5 \mathrm{p}$ and oxygen $2 \mathrm{p}$ with smaller contributions from bismuth $6 \mathrm{~s}$ and $6 \mathrm{p}$ orbitals while the conduction band is made up of bismuth $6 \mathrm{p}$, iodine $5 \mathrm{~s}$ and oxygen $2 \mathrm{p}$ orbitals. As the top of the valence band is derived mainly from iodine $5 p$ orbitals, loss of iodine from BiOI will deplete the density of states there, thus resulting in a widening of the band gap. 
Table 3 Physical and photocatalytic properties of calcined BiOl-pH6

\begin{tabular}{|c|c|c|c|c|c|c|c|c|c|}
\hline Entry & BiOI (\%) & $\mathrm{Bi}_{4} \mathrm{O}_{5} \mathrm{I}_{2}(\%)$ & $\mathrm{Bi}_{7} \mathrm{O}_{9} \mathrm{I}_{3}(\%)$ & $\alpha-\mathrm{Bi}_{5} \mathrm{O}_{7} \mathrm{I}(\%)$ & $A_{\mathrm{BET}}\left(\mathrm{m}^{2} \mathrm{~g}^{-1}\right)$ & $E_{\mathrm{g}}(\mathrm{eV})$ & $C_{\mathrm{ads}}\left(\mathrm{mg} \mathrm{m}^{-2}\right)$ & $k_{\mathrm{W}}\left(\mathrm{h}^{-1} \mathrm{~g}^{-1}\right)$ & $k_{\mathrm{SA}}\left(\mathrm{h}^{-1} \mathrm{~m}^{-2}\right)$ \\
\hline As-syn & 100 & 0 & 0 & 0 & 15.3 & 1.90 & 0.05 & 3.65 & 0.24 \\
\hline $300-1$ & 45 & 55 & 0 & 0 & 30.9 & 2.04 & 0.11 & 6.14 & 0.20 \\
\hline $350-1$ & 0 & 0 & 100 & 0 & 23.5 & 2.56 & 0.05 & 10.6 & 0.45 \\
\hline $350-3$ & 0 & 0 & 65 & 35 & 20.1 & 2.58 & 0.05 & 17.2 & 0.86 \\
\hline $350-5$ & 0 & 0 & 26 & 74 & 17.9 & 2.58 & 0.08 & 14.4 & 0.81 \\
\hline $400-1$ & 0 & 0 & 0 & 100 & 12.8 & 3.16 & 0.10 & 9.22 & 0.72 \\
\hline
\end{tabular}
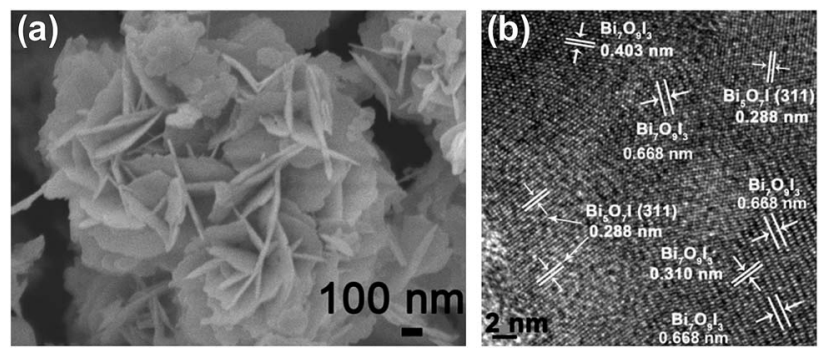

Fig. 9 (a) FESEM and (b) HRTEM images of BiOl-pH6-350-3.

\subsection{Photocatalytic activity of bismuth oxyiodide composites}

Compared to the as-prepared BiOI-pH6, the calcined samples showed up to fivefold higher photocatalytic activity for the degradation of $p$-cresol (Fig. 10). The most active photocatalysts were composites of $\mathrm{Bi}_{7} \mathrm{O}_{9} \mathrm{I}_{3} / \alpha-\mathrm{Bi}_{5} \mathrm{O}_{7} \mathrm{I}$ where $>99 \%$ degradation efficiency was reached after only $2 \mathrm{~h}$. For benchmarking, the degradation was only $37 \%$ over P25 titania. The most active composite has a rate constant, $k_{\mathrm{SA}}$ of $0.86 \mathrm{~h}^{-1} \mathrm{~m}^{-2}$ which is twice that of the $\{001\}$-facetted BiOI. The total organic carbon decreased from $18.7 \mathrm{ppm}$ to $6.14 \mathrm{ppm}$ within $2 \mathrm{~h}$ (Fig. S10†).

The improved activity of the composites can be rationalized in terms of the energy levels of the components. The valence band energy level can be estimated according to: $E_{\mathrm{VB}}=\chi-E^{\mathrm{e}}+$ $(1 / 2) E_{\mathrm{g}}$ where $\chi$, the electronegativity of the semiconductor, is calculated as the geometric mean of the Mulliken electronegativities of the components in the semiconductor, $E^{\mathrm{e}}$ is the energy of free electrons on the hydrogen scale (taken as $4.5 \mathrm{eV}$ ), and $E_{\mathrm{g}}$ is the band gap of the semiconductor as determined

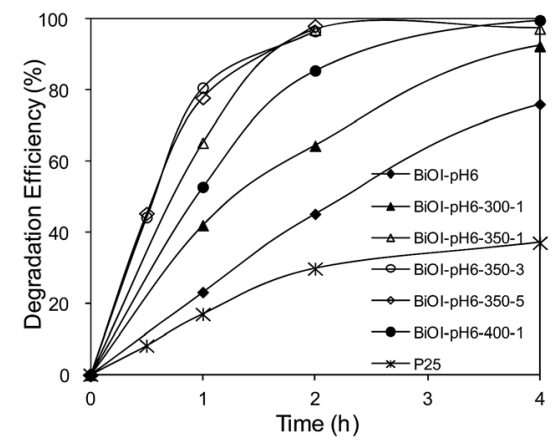

Fig. 10 Effect of calcination on photocatalytic activity of BiOl-pH6 and P25. from the onset of the optical transition (Fig. S11†). ${ }^{30}$ In the $\mathrm{Bi}_{7} \mathrm{O}_{9} \mathrm{I}_{3} / \alpha-\mathrm{Bi}_{5} \mathrm{O}_{7} \mathrm{I}$ system, the conduction band (CB) and valence band (VB) of $\mathrm{Bi}_{7} \mathrm{O}_{9} \mathrm{I}_{3}$ are more negative than those of $\alpha-\mathrm{Bi}_{5} \mathrm{O}_{7} \mathrm{I}$, thus the photo-generated electrons can transfer from the $\mathrm{CB}$ of $\mathrm{Bi}_{7} \mathrm{O}_{9} \mathrm{I}$ into that of $\alpha-\mathrm{Bi}_{5} \mathrm{O}_{7} \mathrm{I}$ whereas holes can move from the VB of $\alpha-\mathrm{Bi}_{5} \mathrm{O}_{7} \mathrm{I}$ to that of $\mathrm{Bi}_{7} \mathrm{O}_{9} \mathrm{I}$ (Fig. 11). The separation of the photoinduced electron-hole pairs at the interface of the two semiconductors increases the lifetime of the electrons and holes.

The composite $\mathrm{Bi}_{7} \mathrm{O}_{9} \mathrm{I}_{3} / \alpha-\mathrm{Bi}_{5} \mathrm{O}_{7} \mathrm{I}$ was tested for photodegradation of other phenolic compounds under visible light irradiation. Substituted phenols containing electron donating alkyl groups, such as $p$-cresol and 4-tert-butylphenol, were more easily degraded than phenol or 4-chlorophenol (Fig. 12). After $2 \mathrm{~h}, \mathrm{p}$-cresol and 4-tert-butylphenol were degraded to more than $99 \%$ whereas the degradation efficiency was only $\sim 39 \%$ and $\sim 50 \%$ for phenol and 4 -chlorophenol, respectively.

After each batch reaction, the catalyst was recovered and recalcined at $300{ }^{\circ} \mathrm{C}$ for $2 \mathrm{~h}$ to remove any adsorbed organics on the surface. The recycled catalyst maintained its photodegradative activity for $p$-cresol even after four runs (Fig. S12a $\dagger$ ). The X-ray diffractograms of the fresh and recycled catalysts showed no significant difference (Fig. S12b $\dagger$ ), indicating its stability and reuseability. Additionally, we also examined the photoactivity in the presence of coexisting ions, which are common in wastewater (Fig. S13 $\dagger$ ). The sample BiOI-pH6-350-3 maintained its activity in the presence of $1 \mathrm{mmol} \mathrm{L}^{-1} \mathrm{NO}_{3}{ }^{-}$and even had a slightly higher activity in the presence of $\mathrm{Cl}^{-}$. These results support the potential usage of the material for wastewater treatment.

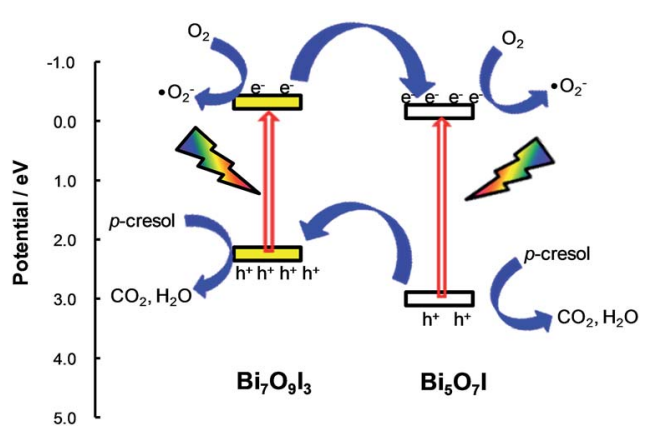

Fig. 11 Energy levels of $\mathrm{Bi}_{7} \mathrm{O}_{9} \mathrm{I}_{3} / \alpha-\mathrm{Bi}_{5} \mathrm{O}_{7} \mathrm{l}$. 


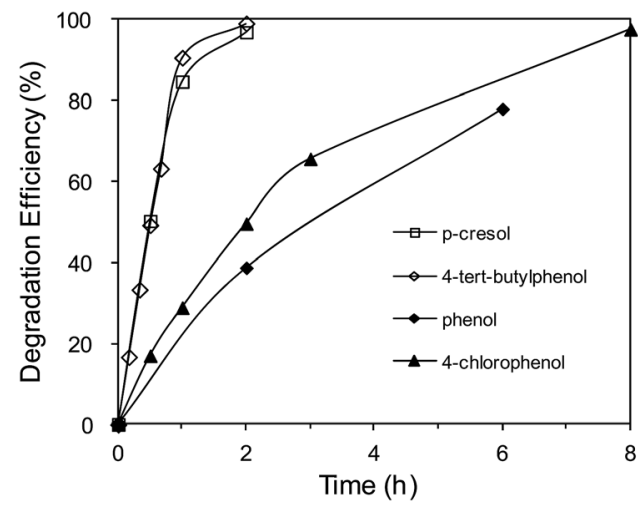

Fig. 12 Photocatalytic activity of BiOl-pH6-350-3 for phenolic compounds.

\subsection{Active species}

In order to gain some insight into the active species involved in the photodegradation of $p$-cresol over $\mathrm{Bi}_{7} \mathrm{O}_{9} \mathrm{I}_{3} / \alpha-\mathrm{Bi}_{5} \mathrm{O}_{7} \mathrm{I}$, radical scavengers and $\mathrm{N}_{2}$ and $\mathrm{O}_{2}$ purging were used. The presence of the ${ }^{\circ} \mathrm{OH}$ radical can be detected by its reaction with terephthalic acid to produce 2-hydroxyterephthalic acid which fluoresces at $425 \mathrm{~nm} \cdot{ }^{31}$ However, there was no obvious increase in the peak intensity at $425 \mathrm{~nm}$ even after $2 \mathrm{~h}$ of irradiation (Fig. S14†), indicating that no ${ }^{\circ} \mathrm{OH}$ radicals were formed for the $\mathrm{Bi}_{7} \mathrm{O}_{9} \mathrm{I}_{3} /$ $\alpha-\mathrm{Bi}_{5} \mathrm{O}_{7} \mathrm{I}$ composite. Addition of excess tert-butanol, a known ${ }^{\circ} \mathrm{OH}$ scavenger, ${ }^{32}$ also did not affect the degradation efficiency (Table 4). The participation of holes was tested by adding a hole scavenger, $\mathrm{K}_{2} \mathrm{C}_{2} \mathrm{O}_{4}$, to the photoreaction system. ${ }^{33}$ The rate constant dropped drastically to only $7 \%$ of that without the scavenger, suggesting that holes play a major role in the degradation of $p$-cresol.

Purging the reaction system with nitrogen to remove any dissolved oxygen led to lower degradation efficiency. In contrast, the reaction rate was increased over that at ambient conditions when oxygen was bubbled into the solution, showing that the presence of oxygen is important. The dissolved oxygen can react with photoinduced electrons to form the superoxide radical ${ }^{\circ} \mathrm{O}_{2}{ }^{-}$or it can act only as an electron acceptor to reduce the electron-hole recombination. To clarify the role of oxygen, $\mathrm{KBrO}_{3}$ was added to the reaction system with and without nitrogen purging. Potassium bromate, a strong oxidant, is

Table 4 Photodegradation of $p$-cresol over $\left.\mathrm{Bi}_{7} \mathrm{O}_{9}\right|_{3} / \alpha-\mathrm{Bi}_{5} \mathrm{O}_{7} \mid$ in the presence of various scavengers and gas atmosphere

\begin{tabular}{lllll}
\hline Scavenger & Purging gas & Amt $(\mu \mathrm{mol})$ & $k\left(\mathrm{~h}^{-1} \mathrm{~g}^{-1}\right)$ & Radical tested \\
\hline- & - & - & 17.2 & - \\
- & $\mathrm{N}_{2}$ & - & 7.20 & $\cdot \mathrm{O}^{-}$ \\
- & $\mathrm{O}_{2}$ & - & 27.9 & $\cdot \mathrm{O}^{-}$ \\
$t$-Butanol & - & 110 & 16.1 & $\cdot \mathrm{OH}$ \\
$\mathrm{K}_{2} \mathrm{C}_{2} \mathrm{O}_{4}$ & - & 110 & 1.19 & $\mathrm{Holes}$ \\
$\mathrm{KBrO}_{3}$ & - & 110 & 122 & $\cdot \mathrm{O}_{2}^{-}$ \\
$\mathrm{KBrO}_{3}\left(\mathrm{~N}_{2}\right)$ & - & 110 & 127 & $\cdot \mathrm{O}_{2}^{-}$ \\
$\mathrm{KBrO}_{3}$ & - & 44 & 102 & $\cdot \mathrm{O}_{2}{ }^{-}$
\end{tabular}

known to be a good electron acceptor and can be reduced as follows: ${ }^{34,35}$

$$
\begin{gathered}
\mathrm{BrO}_{3}^{-}+\mathrm{e}^{-}+2 \mathrm{H}^{+} \rightarrow \mathrm{BrO}_{2}+\mathrm{H}_{2} \mathrm{O} \\
\mathrm{BrO}_{3}^{-}+6 \mathrm{e}^{-}+6 \mathrm{H}^{+} \rightarrow\left[\mathrm{BrO}_{2}^{-}, \mathrm{HOBr}\right] \rightarrow \mathrm{Br}^{-}+3 \mathrm{H}_{2} \mathrm{O}
\end{gathered}
$$

Its addition should reduce the electron-hole recombination in the photocatalyst, thus increasing the quantum efficiency without producing superoxide radicals. The reaction was $\sim 7$ times faster after adding $\mathrm{KBrO}_{3}$ which can be attributed to the decrease of electron-hole recombination. The reaction rate remained high and was unaffected by nitrogen purging, indicating that superoxide radicals are not involved in the degradation process. Rather, the addition of $\mathrm{KBrO}_{3}$ effectively suppressed the recombination of photo-generated holes with electrons resulting in the enhanced activity. This result lends further support that holes are the main species for the photodegradation of $p$-cresol over $\mathrm{Bi}_{7} \mathrm{O}_{9} \mathrm{I}_{3} / \alpha-\mathrm{Bi}_{5} \mathrm{O}_{7} \mathrm{I}$.

Intermediates formed during photodegradation of $p$-cresol 1 were identified using LC-MS (Fig. S15 $\dagger$ ). Several intermediates could be detected. Oxidation of the methyl group in $p$-cresol leads to 4-hydroxybenzyl alcohol 2 which undergoes further reaction to 4-hydroxybenzaldehyde 4 and 4-hydroxybenzoic acid 6 (Scheme 1). Dimerization of 4-hydroxybenzyl radical gives 4-(4-hydroxy phenethyl)phenol 7. Its formation has been attributed to the relatively long lifetime of the 4hydroxybenzyl radical intermediate, which increases the probability of dimerization. ${ }^{36}$ Besides oxidation at the methyl group, products due to the benzene ring oxidation such as 4methyl benzene diols 3 and dihydroxy benzaldehydes 5 were also detected. Mineralization of the intermediates into $\mathrm{CO}_{2}$ and $\mathrm{H}_{2} \mathrm{O}$ was confirmed by the decrease in the TOC upon photoirradiation.

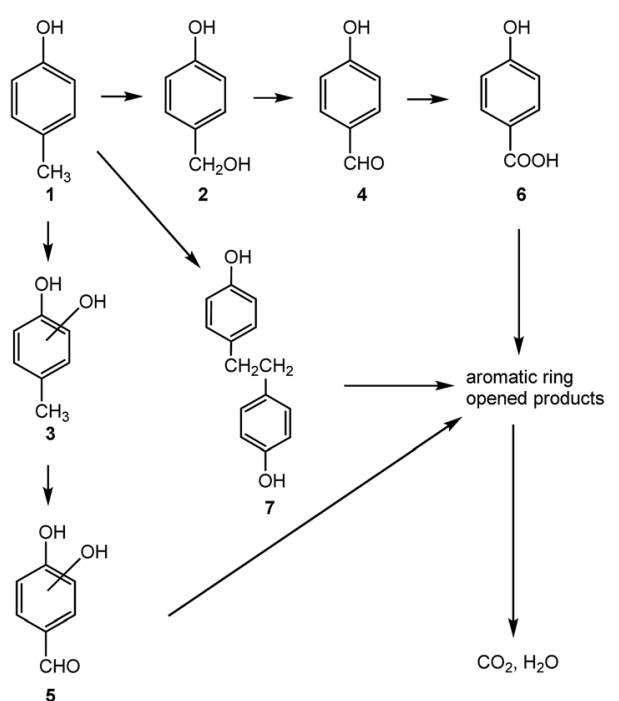

Scheme 1 Proposed degradation pathway of $p$-cresol over BiOlpH6-350-3. 


\section{Conclusions}

Single crystalline nanosheets of BiOI with either predominantly $\{001\}$ - or $\{110\}$-exposed facets were obtained by varying the $\mathrm{pH}$ during hydrothermal synthesis. Under acidic conditions $\mathrm{pH}$ 2.3-4), the $\{001\}$ facets were preferentially formed while BiOI with $\{110\}$ dominant facets was formed at pH 5 to 8 . The surface area of BiOI increased with increasing synthesis $\mathrm{pH}$ due to the formation of smaller and thinner nanosheets. The intrinsic photoactivity for $p$-cresol degradation under visible light irradiation of the $\{001\}$-facetted BiOI was about twice that of BiOI with $\{110\}$ exposed facets when compared on unit surface area. Calcination of the $\{110\}$-facetted BiOI led to the replacement of some of the iodine by oxygen. The heterojunctioned $\mathrm{Bi}_{x} \mathrm{O}_{y} \mathrm{I}_{z}$ composites formed by this process showed better photocatalytic activity than $\mathrm{BiOI}$. $\mathrm{A} \mathrm{Bi}_{7} \mathrm{O}_{9} \mathrm{I}_{3} / \alpha-\mathrm{Bi}_{5} \mathrm{O}_{7} \mathrm{I}$ composite had the highest photocatalytic activity for $p$-cresol and other phenolic compounds. Experiments with various scavengers and under aerobic and anaerobic conditions point to holes as the species responsible for most of the photodegradation.

\section{Acknowledgements}

Financial support from the National Research Foundation and the Economic Development Board (SPORE, COY-15-EWIRCFSA/N197-1) for a research assistantship for A. Han and a research scholarship for J. Sun is gratefully acknowledged. The work is supported by the Ministry of Education ARC Tier 1 grants R-143-000-550-112 and R-143-000-603-112.

\section{References}

1 R. Asahi, T. Morikawa, T. Ohwaki, K. Aoki and Y. Taga, Science, 2001, 293, 269-271.

2 J. G. Yu, Y. R. Su and B. Cheng, Adv. Funct. Mater., 2007, 17, 1984-1990.

3 Z. Zhang, C. C. Wang, R. Zakaria and J. Y. Ying, J. Phys. Chem. $B$, 1998, 102, 10871-10878.

4 W. Chen, Q. Kuang, Q. Wang and Z. Xie, RSC Adv., 2015, 5, 20396-20409.

5 J. N. Wilson and H. Idriss, J. Am. Chem. Soc., 2002, 124, 11284-11285.

6 M. Lazzeri, A. Vittadini and A. Selloni, Phys. Rev. B: Condens. Matter Mater. Phys., 2001, 63, 155409.

7 J. Jiang, K. Zhao, X. Xiao and L. S. Zhang, J. Am. Chem. Soc., 2012, 134, 4473-4476.

8 R. Li, F. Zhang, D. Wang, J. Yang, M. Li, J. Zhu, X. Zhou, H. Han and C. Li, Nat. Commun., 2013, 4, 1432.

9 Y. Yang, G. Wang, Q. Deng, H. Wang, Y. Zhang, D. H. L. Ng and H. Zhao, RSC Adv., 2014, 4, 34577-34583.
10 Y. Bi, S. Ouyang, N. Umezawa, J. Cao and J. Ye, J. Am. Chem. Soc., 2011, 133, 6490-6492.

11 J. Miao and B. Liu, RSC Adv., 2013, 3, 1222-1226.

12 H. Zhang, L. Liu and Z. Zhou, RSC Adv., 2012, 2, 9224-9229.

13 J. Henle, P. Simon, A. Frenzel, S. Scholz and S. Kaskel, Chem. Mater., 2007, 19, 366-373.

14 Z. Liu, W. Xu, J. Fang, X. Xu, S. Wu, X. Zhu and Z. Chen, Appl. Surf. Sci., 2012, 259, 441-447.

15 P. Q. Wang, Y. Bai, J. Y. Liu, Z. Fan and Y. Q. Hu, Micro Nano Lett., 2013, 8, 99-101.

16 Y. Wang, K. Deng and L. Zhang, J. Phys. Chem. C, 2011, 115, 14300-14308.

17 X. Chang, J. Huang, Q. Tan, M. Wang, G. Ji, S. Deng and G. Yu, Catal. Commun., 2009, 10, 1957-1961.

18 R. Hao, X. Xiao, X. Zuo, J. Nan and W. Zhang, J. Hazard. Mater., 2012, 209-210, 137-145.

19 X. Wang, S. Yang, H. Li, W. Zhao, C. Sun and H. He, RSC Adv., 2014, 4, 42530-42537.

20 X. Xiao and W. Zhang, J. Mater. Chem., 2010, 20, 5866-5870. 21 J. Liu, H. Li, N. Du, S. Song and W. Hou, RSC Adv., 2014, 4, 31393-31399.

22 L. Ye, L. Tian, T. Peng and L. Zan, J. Mater. Chem., 2011, 21, 12479-12484.

23 E. Keller and V. Krämer, Acta Crystallogr., Sect. C: Cryst. Struct. Commun., 2007, C63, i109-i111.

24 P. Rittner and H. Oppermann, Z. Anorg. Allg. Chem., 1992, 617, 131-135.

25 M. Schmidt, H. Oppermann, H. Brückner and M. Binnewies, Z. Anorg. Allg. Chem., 1997, 623, 1945-1953.

26 E. Keller, V. Krämer, M. Schmidt and H. Oppermann, Z. Kristallogr.-Cryst. Mater., 2002, 217, 256-264.

27 U. Eggenweiler, J. Ketterer, E. Keller and V. Krämer, Z. Kristallogr.-Cryst. Mater., 2001, 216, 230-233.

28 J. Ketterer, E. Keller and V. Krämer, Z. Kristallogr.-Cryst. Mater., 1985, 172, 63-70.

29 R. A. Smith, Semiconductors, Cambridge University Press, Cambridge, U.K., 2nd edn, 1978.

30 M. A. Butler and D. S. Ginley, J. Electrochem. Soc., 1978, 125, 228-232.

31 C. Karunakaran and P. Anilkumar, J. Mol. Catal. A: Chem., 2007, 265, 153-158.

32 J. Ma and N. J. D. Graham, Water Res., 2000, 34, 3822-3828. 33 A. J. Hoffman, E. R. Carraway and M. R. Hoffmann, Environ. Sci. Technol., 1994, 28, 776-785.

34 S. Qourzal, N. Barka, M. Tamimi, A. Assabbane and Y. AitIchou, Appl. Catal., A, 2008, 334, 386-393.

35 W. Bahnemann, M. Muneer and M. M. Haque, Catal. Today, 2007, 124, 133-148.

36 A. Mylonas and E. Papaconstantinou, Polyhedron, 1996, 15, 3211-3217. 